\title{
Kata Kolok Color Terms and the Emergence of Lexical Signs in Rural Signing Communities
}

\section{Connie de Vos}

\section{ABSTRACT How do new languages} develop systematic ways to talk about sensory experiences, such as color? To what extent is the evolution of color terms guided by societal factors? This article describes the color lexicon of a rural sign language called Kata Kolok that emerged approximately one and a half centuries ago in a Balinese village. Kata Kolok has four color signs: black, white, red, and a blue-green term. In addition, two non-conventionalized means are used to provide color descriptions: naming relevant objects, and pointing to objects in the vicinity. Comparison with Balinese culture and spoken Balinese brings to light discrepancies between the systems, suggesting that neither cultural practices nor

\author{
Connie de Vos' \\ research at the Max \\ Planck Institute for \\ Psycholinguistics \\ focuses on spatial \\ language in Kata \\ Kolok, a rural sign \\ language of North-Bali. \\ clgdevos@uclan.ac.uk
}


of color signs in Kata Kolok. The few lexicographic investigations from other rural sign languages report limitations in the domain of color. On the other hand, larger, urban signed languages have extensive systems, for example, Australian Sign Language has up to nine color terms. These comparisons support the finding that rural sign languages like Kata Kolok fail to provide the societal pressures for the lexicon to expand further.

KEYWORDS: Kata Kolok, sign language, language of perception, color, iconicity, semantic categories

\section{The Kata Kolok Community}

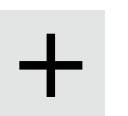

Kata Kolok is used in a village of North Bali which is home to fifty deaf individuals of varying ages in a population of 2,200 . This incidence of deafness is extremely high compared to numbers found in, for instance, the USA where 1 in 1,000-2,000 children is born with a severe hearing impairment (Morton 1991). Deafness in the village stems from a recessive gene resulting in shortened hair cells in the cochlea. Anthropological and genetic research reveals that the sudden increase of deafness that led to the emergence of Kata Kolok took place up to twelve generations ago (Winata et al. 1995: 342-3).

Unlike the signed languages used in urban societies, most hearing people in the Kata Kolok community are accustomed to signing in interaction with deaf members of the community. Deaf villagers are integrated into the wider hearing community, as evidenced by their occupational and marital status (Branson et al. 2002: 125-8). That is, deaf people have similar occupations, and similar chances of marrying as hearing villagers do. The signed language is part of several cultural adaptations to deafness, which also include unique ceremonial tasks in the burying of the dead, belief in a deaf God, and a specialized dance performed by the deaf villagers (Marsaja 2008: 75).

The type of community outlined above is usually referred to as a "deaf village" and although it is exceptional, it is not unique. There are reports of deaf villages and associated rural sign languages from around the globe. Rural sign languages are usually contrasted with the urban sign languages of national deaf communities. Rural sign languages are used in small communities (around 1,000-2,000 people) with only a small proportion of deaf signers (less than 10 percent). Signers are in close daily contact with each other, and the language is not normally used in formal deaf education. Conversely, urban sign languages find their origin in the formation of deaf schools and may have as many as 1.5 million signers (Indo-Pakistani Sign Language; Zeshan 2000). Both urban sign languages and rural sign 
languages may arise from scratch; nevertheless, most rural sign languages are relatively recent in origin while the histories of urban sign languages can often be traced back to the set-up of formal deaf education or other known signed languages further back in time. It should be noted that these characteristics are generalizations, and that individual signing communities may deviate from the overall pattern.

One of the major findings of linguistics in the past half century is that signed languages are of equal linguistic complexity to spoken languages. That is, languages of both the visual and auditory modality have conventionalized vocabularies, are governed by syntactic structure, and show similar timetables for acquisition (Meier et al. 2002). Although rural sign languages were not included in sign research until recently, there are no indications that these languages are either communicatively or linguistically less complex. Kata Kolok is used in all aspects of village life ranging from village politics, Hindu ceremonies, gossip, and education. It even has a specialized register used in child-directed signing. The first descriptions of the language have revealed complex structures in all main linguistic domains (Marsaja 2008). In every sense Kata Kolok should be taken to be a fully-fledged language.

\section{Data Collection}

This article and wider research by the author are based on longterm language documentation. For the elicitation of color terms, a standardized stimulus was used that consisted of eighty color chips from the Munsell color chart (see Majid and Levinson 2007). Eight profoundly deaf, native signers of Kata Kolok were filmed as they provided descriptions for each of these chips. For ethnographic information of the Balinese culture and language I have relied on my own observations in the field as well as conversations with two hearing Balinese informants: Ketut Kanta (who is from the village) and Made Hery Santosa who is from a nearby city (Singaraja).

\section{Kata Kolok Color Descriptions}

Kata Kolok has four terms to describe colors: "white," "black," "red," and "grue," i.e. a term covering both English blue and green colors. The sign for "white" is made by pointing at one's teeth, and can also be taken to mean teeth depending on sentential context. The sign for "black" is made by briefly rubbing a fraction of one's hair between thumb and index finger. The sign can also refer to hair. Like the sign for "white/teeth," the meaning of "black/hair" is dependent on the context of the utterance. "Red" is formed by slowly stroking the index finger across the lips. It cannot refer to lips, but the meaning is often extended to refer to the (red) 100,000 Rupiah note. All three signs are iconic in the sense that their forms are in part representative of their meanings. Finally, the sign "grue" is made by sweeping the hand across the forehead three times. Signers do not attribute an 


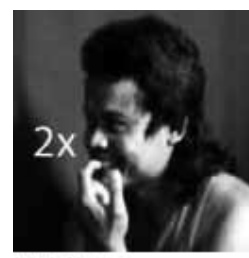

WHITE

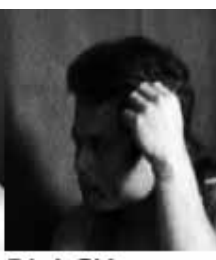

BLACK

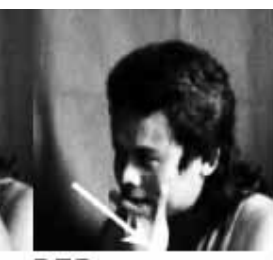

RED

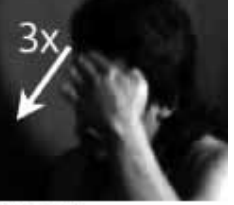

GRUE

iconic origin to the sign. Moreover, it is the only sign exclusively used for color descriptions. The four color signs are illustrated by stills in Figure 1.

The lexical status of these four terms becomes apparent from their integration into the linguistic system as evidenced by their co-occurrence with an intensifier (e.g. "very black"). The intensifier is formed by repetition and enlargement of the sign as well as pursed lips, squinted eyes, and a wrinkled nose simultaneous with the lexical sign. Intensification of the signs for "white" and "red" lacks the pursed lips marker presumably for reasons of production, i.e. since these signs are produced at the mouth, pursed lips would interfere with the production of the manual sign. As the "grue" sign has inherent repetition of movement, the intensified form is thus identified by enlargement and the use of facial expressions. In Figure 1, the "grue" example illustrates the pursed lips of the intensity marker.

The four described color signs are conventional in the sense that they are known and used by all participants. The four color signs form non-contiguous categories and do not exhaust all colors in the spectrum. Or to put it another way: there are lexical gaps. Kata Kolok can thus be classified as a non-partitioning language (Kay and Maffi 1999: 746; Hill, this issue). Despite the lack of lexicalized color signs, many color chips received color descriptions that have highly context-dependent forms. Two strategies occur in the data: naming an object that typically has the color of the presented color patch (e.g. banana to indicate yellow) and pointing at an object within the vicinity that is colored in the same way, e.g. a piece of clothing. The interpretation of an object as a color description is dependent on sentential context. These two forms are framed as color descriptors by ostensive searching behavior of the signer before they occur. Notably, these strategies may be used to form ad-hoc categories in which e.g. a blue chair can represent various colors within the blue-green spectrum, and a wooden door can represent brown colors. Object naming and pointing are the most frequent strategies found in the data, and most signers stuck with one strategy during the elicitation session. Crucially, unlike the four lexical color terms, the two non-conventionalized types of structures cannot be used with the intensifier.

The fact that all signers have these non-conventionalized color descriptors at hand raises the question of why Kata Kolok has

\section{Figure 1}

Kata Kolok's four color signs: white, black, red, and grue (blue-green). 
developed lexical signs at all. In my view, the answer lies in the semiotic and formal properties of these signs. Like the non-lexical way of describing color by pointing at objects in the vicinity, the explicit reference to body parts in the case of white/teeth and black/ hair as well as the implicit reference to lips in the sign for red are based on iconic-indexicality. That is, an exemplar that resembles the described object with respect to its color properties is used as a stand-in. Similarly, in the case of non-lexical pointing for color the signs are dependent on the objects available within visual range. Since the body provides a fixed context for signers however, the indication of body parts may have become easily ritualized.

The sign for grue remains unique in the sense that no signer currently attributes an iconic meaning to it. Looking at both the semiotic and formal properties of the sign, two possible sources are suggested. First, it might be related to the signs for bushes or sea that involve the same hand shape and a triple repeated waving gesture on the signer's lateral axis. Although the semantics are similar, I find the following possibility more likely. That is, the grue sign may be historically related to the act of putting wet rice (bija) on the forehead at Hindu ceremonies. The sign in reference to this practice involves the same hand shape, the same place of articulation (the forehead), and a threefold repetition of touching the forehead. In rare instances the rice used in this ritual is colored yellow by the use of turmeric. This sign for bija could be the source because of the similar formal properties of the sign grue. That is, it would require only the type of movement to change. If the current sign for grue was derived from the water or bushes sign this would require the alteration of more phonological parameters, i.e. type of movement, orientation, and place of articulation would also have had to change. The latter change in particular is quite unlikely as the forehead is a rare place of articulation in Kata Kolok and in signed languages in general, and signs actually tend to move away from this place of articulation (see Nonaka 2004 for a summary of phonological change in signed languages). Historically this grue sign may have covered the blue/ green/yellow domain, but today it is more limited because of several competing objects that signers use to describe yellow colors. These objects include a sign making reference to turmeric directly, and the sign for banana. As predicted by Kay (1975: 263) these emergent and competing terms show more variability among signers. Nevertheless, these non-basic color terms may influence the category boundaries of the basic proto-grue term, as has previously been documented for the non-partitioning language Yélî Dnye (Levinson 2000: 26). The inference of an intermediate system with a term that was formally related to the baji sign and the current grue term but with a blue/ green/yellow meaning is a developmental path also described by Kay and Maffi (1999: 753). 


\section{The Salience of Color in Balinese Culture}

Cultural practices are often thought to be the main driving force behind color lexicalization (Levinson 2000). The comparative simplicity of Kata Kolok's color system may lead to the hypothesis that contemporary Balinese culture deemphasizes color and has thus prevented the development of an elaborate color system in the sign language. Nothing seems to be further from the truth. For Balinese people, color words are part of a cultural complex associating color with specific Gods, cardinal directions, and body parts. What is more, both the deaf and hearing members of the Kata Kolok community exercise shared religious practices involving food-dyeing in offers for the Gods based on these associations. Deaf and hearing women collaborate in the preparation of colored food offerings at numerous Hindu ceremonies throughout the liturgical year. Data from the Kata Kolok community gives us the chance to review linguistic data from a community that shares one culture but has two fundamentally different modes of linguistic expression - visual-gestural and oral-aural. It might come as a surprise then that spoken Balinese has more than double the number of distinct color terms in comparison to Kata Kolok. The Balinese terms are selem 'black,' putih 'white,' barak/bang 'red,' kuning 'yellow,' pelung 'blue,' gadang 'green,' ungu 'purple,' soklat 'brown, chocolate color,' brumbun 'multicolored,' kelawu 'gray,' and orenz 'orange.'

The differences between both systems are not limited to size. Unlike the Kata Kolok signs for "white" and "black," the Balinese words cannot refer directly to body parts. And although the spoken Balinese color terms are indirectly associated with cardinal directions and thus certain body parts as part of a wider cultural construct (Covarrubias 1950: 88, 296), these associations do not correspond to the body parts indicated in the Kata Kolok signs. Moreover, one should also note that in contrast to Kata Kolok, spoken Balinese strictly divides blues and greens into separate categories. All in all, it is highly unlikely that either spoken Balinese or Balinese culture is a direct source for the Kata Kolok color term system. The differences between the Kata Kolok and Balinese linguistic systems make clear that it is possible to share a culture across two radically different languages, one of which has less color words and less restrictive categories in this particular perceptual domain.

\section{The Lexica of Rural Sign Languages}

A lesser degree of lexicalization in the color domain has also been observed in other rural sign languages such Adamorobe Sign Language of Ghana (Nyst 2007), Al-Sayyed Bedouin Sign Language of Israel (Wendy Sandler, personal communication, August 26, 2010), Ban Khor Sign Language of Thailand (Nonaka 2004), and Providence Island Sign Language (Woodward 1989). These sign languages all have limited systems that do not exceed three color terms. Moreover, the non-lexical, alternative strategy of pointing 
for color is also reported for each of these sign languages. What is more, in each case, it is neither the surrounding spoken language, nor the wider hearing culture that fully explain the lexical limitations of these systems. Given that there are many rural spoken language communities with elaborate color term systems it is probable that the visual-gestural language modality also plays a role in the observed patterns. Nonetheless, the comparison to urban sign languages raises the question of whether there is something special about rural sign languages that prevents them from expanding their color terminologies.

If the lexica of rural sign languages are indeed less pressured to develop lexical conventions for perceptual categories, this phenomenon should not be restricted to color terms nor perceptual experience per se. One would expect similar effects to be found in other domains of the lexicon as well, and this is indeed the case. What reports we do have of lexica of rural sign languages do indeed reveal further convergence. Both Kata Kolok and Providence Sign Language signs have been reported to have highly contextdependent meanings (Washabaugh et al. 1978; Marsaja 2008). Moreover, the kinship systems of Kata Kolok and Adamorobe Sign Language (Nyst 2007: 100) are limited in having only terms for grandparent, mother, father, and offspring. The Providence Island Sign Language system appears to be even more restricted with its three kinship terms for mother, father, and offspring (Woodward 1978: 128). All three signed languages use the sign meaning same to indicate non-lineal relatives. Although more extensive comparative research is needed, these findings are suggestive of the hypothesis that the lexica of rural sign languages may be limited all-round.

These observations raise the question of what causes the relatively limited set of lexical expressions in what is otherwise a communicatively fully adept system. Or put differently, what societal factors have resulted in the high degree of lexical proliferation and systematicity in the case of urban sign languages such as Australian Sign Language (see Woodward 1989: 149)? The lack of spatial verb agreement found in the rural sign languages Al-Sayyed Beduin Sign Language has been attributed to its relatively limited time depth (Sandler et al. 2005: 2665). Could time depth be responsible for the lack of lexical proliferation in the color terminologies of rural sign languages as well? Although time depth is certainly a prerequisite for development, a comparison of the color lexica of the rural sign language Adamorobe Sign Language - estimated to be 200 years old - which has three lexical color signs (Nyst 2007: 25) and Israeli Sign Language - estimated to be 75 years old - which has seventeen color signs for fifteen colors (Meir and Sandler 2008; Sara Lanesman, personal communication, August 26, 2010) indicates that time by itself is unlikely to be a driving force behind lexicalization.

In my view, the comparison of rural and urban sign languages suggests that the communicative settings in which rural sign 
languages are used may not require signers to fully lexicalize perceptual experiences such as color. What might be the societal processes that give rise to this effect? First of all, given the fact that signed conversations necessarily take place in face-to-face interaction, one may resort to pointing as a strategy for color description. In a way, signs need not be portable or context-independent, as they are rarely used in detached situations. Secondly, since rural sign languages are used in small, isolated communities, villagers are aware of each other's idiosyncratic expressions. For this reason, more variability in signed forms to refer to color may be permitted. Finally, unlike urban sign languages, rural sign languages are not normally used in formal deaf education, which might accelerate the calibration of color signs to the color words of the surrounding spoken language. That is, unlike urban sign languages, Kata Kolok has not adopted the surrounding Balinese system, despite a cultural preoccupation with color in everyday life. It thus seems that the manifestation of signed communication may determine the degree to which the lexical conventionalization takes place. As such, the development of village sign lexica in their social habitats may broaden our understanding of the coevolution and calibration of cultural and communicative practices.

\section{Acknowledgments}

This study was carried out as part of the Language of Perception study within the Categories across Language and Cognition project at the Max Planck Institute for Psycholinguistics. I would like to thank all the Balinese participants in this study, in particular Ketut Kanta and Made Hery Santosa for sharing their insights in the Balinese language and culture. I would also like to thank Sara Lanesman and Wendy Sandler with their help on data from Israeli Sign Language and Al-Sayyed Bedouin Sign Language. Finally, I would like to thank Stephen C. Levinson, Asifa Majid, Nick Palfreyman, and Pamela Perniss for their comments on earlier drafts of the article. The research reported here was supported by the Max Planck Gesellschaft.

\section{References}

Branson, Jan, Miller, Don and Marsaja, I Gede. 2002. "Sign Languages as a Natural Part of the Linguistic Mosaic: The Impact of Deaf People on Discourse Forms in North Bali, Indonesia." In Elizabeth Winston (ed.), Storytelling and Conversation Discourse in Deaf Communities, pp. 109-48. Washington, DC: Gallaudet University Press.

Covarrubias, Miguel. 1950. Island of Bali. New York: Alfred A. Knopf. Kay, Paul. 1975. "Synchronic Variability and Diachronic Change in Basic Color Terms." Language in Society 4(3): 257-70.

Kay, Paul and Maffi, Luisa. 1999. "Color Appearance and the Emergence and Evolution of Basic Color Terms." American Anthropologist 101(4): 743-60. 
Levinson, Stephen C. 2000. "Yélî Dnye and the Theory of Basic Color Terms." Journal of Linguistic Anthropology 10(1): 3-55.

Majid, Asifa and Levinson, Stephen C. 2007. "The Language of Vision I: Colour." In Asifa Majid (ed.), Field Manual, Vol. 10. Nijmegen: Max Planck Institute for Psycholinguistics.

Marsaja, I Gede. 2008. Desa Kolok - A Deaf Village and Its Sign Language in Bali, Indonesia. Nijmegen: Ishara Press.

Meier, Richard, Cormier, Kearsy and Quinto-Pozos, David. 2002. Modality and Structure in Signed and Spoken Language. Cambridge: Cambridge University Press.

Meir, Irit and Wendy Sandler. 2008. A Language in Space: The Story of Israeli Sign Language. New York: Lawrence Erlbaum Associates.

Morton, Newton. 1991. "Genetic Epidemiology of Hearing Impairment." Annals of the New York Academy of Sciences 630(1): 16-31.

Nonaka, Angela. 2004. "The Forgotten Endangered Languages: Lessons on the Importance of Remembering from Thailand's Ban Khor Sign Language." Language and Society 33(5): 737-67.

Nyst, Victoria. 2007. "A Descriptive Analysis of Adamorobe Sign Language (Ghana)." Ph.D. thesis, University of Amsterdam.

Sandler, Wendy, Meir, Irit, Padden, Carol and Aronoff, Mark. 2005. "The Emergence of Grammar: Systematic Structure in a New Language." Proceedings of the National Academy of Sciences 102(7): 2661-5.

Washabaugh, Wiliam, Woodward, James and DeSantis, Susan. 1978. "Providence Island Sign: A Context-Dependent Language." Anthropological Linguistics 20(3): 96-107.

Winata, Sunaryana, Arhya, I Nyoman, Moeljopawiro, Sugonio, Hinnant, John, Liang, Yong, Friedman, Thomas and Asher, James. 1995. "Congenital Non-Syndromal Autosomal Recessive Deafness in Bengkala, an Isolated Balinese Village." Journal of Medical Genetics 32(5): 336-43.

Woodward, James. 1978. "All in the Family: Kinship Lexicalization across Sign Languages." Sign Language Studies 19(4): 121-38.

Woodward, James. 1989. "Basic Color Term Lexicalization across Sign Languages.” Sign Language Studies 63(4): 145-52.

Zeshan, Ulrike. 2000. Sign Language in Indo-Pakistan. Amsterdam: John Benjamins. 